\title{
Reaction mechanisms in collisions induced by halo and weakly bound nuclei around the Coulomb barrier
}

\author{
V.Scuderi ${ }^{1,2,3^{*}}$, A.Di Pietro ${ }^{2}$, L.Acosta ${ }^{4}$, F.Amorini ${ }^{1,2}$, M.J.G.Borge ${ }^{5}$, P.Figuera ${ }^{2}$, \\ M.Fisichella $^{1,2}$, L.M.Fraile ${ }^{6 \dagger}$, J.Gomez-Camacho ${ }^{7}$, H.Jeppesen ${ }^{6}$, M.Lattuada ${ }^{1,2}$, \\ I.Martel $^{4}$, M.Milin ${ }^{8}$, A.Musumarra ${ }^{2,9}$, M.Papa ${ }^{2}$, M.G.Pellegriti ${ }^{1,2}$, F.Perez-Bernal ${ }^{4}$, \\ R.Raabe $^{10}$, G.Randisi ${ }^{1,2}$, F.Rizzo $^{1,2}$, D.Santonocito ${ }^{2}$, G.Scalia $^{1,2}$, O.Tengblad $^{5}$, \\ D.Torresi $^{1,2}$, A.M.Vidal $^{5}$, M.Zadro $^{11}$ \\ ${ }^{I}$ Dipartimento di Fisica ed Astronomia Università di Catania, Catania, Italy \\ ${ }^{2}$ INFN-Laboratori Nazionali del Sud and Sezione di Catania, Italy \\ ${ }^{3}$ Centro Siciliano di Fisica Nucleare e Struttura della Materia, Catania, Italy \\ ${ }^{4}$ Departamento de Fisica Aplicada Universidad de Huelva, Huelva, Spain \\ ${ }^{5}$ Instituto de Estructura de la Materia CSIC, Madrid, Spain \\ ${ }^{6}$ CERN, Geneva, Switzerland \\ ${ }^{7}$ Departamento de Fisica Atomica Molecular Nuclear Universidad de Sevilla, Sevilla, Spain \\ ${ }^{8}$ Department of Physics Faculty of Science University of Zagreb, Zagreb, Croatia \\ ${ }^{9}$ Dipartimento di Metodologie Fisiche e Chimiche per l'Ingegneria Università di Catania, \\ Catania, Italy \\ ${ }^{10}$ Instituut voor Kern-en Stralingsfysica University of Leuven, Leuven, Belgium \\ ${ }^{11}$ Division of Experimental Physics Ruder Bo·skovic Institute, Zagreb, Croatia \\ e-mail: scuderiv@lns.infn.it
}

Elastic scattering and direct reactions have been studied for the collisions induced by the three Beryllium isotopes ${ }^{9,10,11} \mathrm{Be}$, on a medium mass ${ }^{64} \mathrm{Zn}$ target at energies around the Coulomb barrier. The elastic scattering angular distributions, measured for the three systems at the same center of mass energy, were analyzed within the Optical Model and reaction cross-sections were deduced from optical model calculations. For the ${ }^{11} \mathrm{Be}$ induced reaction the transfer/break-up angular distribution was also extracted.

${ }^{1}$ XLVIII International Winter Meeting on Nuclear Physics - BORMIO2010 Bormio, Italy

January 25-29 2

*Speaker

†present address: Departamento de Fysica Atomica, Molecular y Nuclear, Universidad Complutense, Madrid, Spain

tpresent address: Nuclear Science Division, Lawrence Berkeley National Laboratory, Berkeley, USA §present address: LPC-ENSICAEN, IN2P3-CNRS and Université de Caen, France 


\section{Introduction}

Elastic scattering and reaction mechanisms in collisions induced by halo nuclei around the Coulomb barrier is a challenging problem in nuclear reaction studies. In fact, in order to proper describe the scattering and the reaction processes accurate description of the projectile halo structure, and the correct coupling to bound and unbound states of the halo nucleus must be considered. Since ${ }^{6} \mathrm{He}$ beam is nowadays available in different facilities with good intensity, up to $10^{7} \mathrm{pps}$, in a wide range of energies, several experiments have been performed so far using mainly this two neutron halo nucleus on different targets [1-5]. A common feature observed in the ${ }^{6} \mathrm{He}$ induced elastic scattering, is a reduction of the cross-section in most of the measured angular range $[2,5]$. Consequently to the reduction of the elastic cross-section, the total reaction (TR) crosssection is found to be much larger, a factor of two in some case, than the one observed in reactions induced by the corresponding well bound isotope [1,2]. Moreover, different papers $[1,2,6]$ have evidenced that the TR cross-section in collision induced by ${ }^{6} \mathrm{He}$ around and below the Coulomb barrier are dominated by direct reaction processes such as transfer and break-up.

Optical Model (OM) calculations [1, 5] have shown that the optical potential of halo and weakly bound nuclei has a long range part due to the coupling to non-elastic channels and that a large diffuseness of the imaginary part of the potential is needed in order to take into account the diffuse structure of the ${ }^{6} \mathrm{He}$ halo and to describe the data. Continuum Discretized Coupled Channel calculations (CDCC) of the ${ }^{6} \mathrm{He}$ elastic scattering on different target masses (e.g. [7, 8]) have been recently published. According to these calculations, the suppression of the rainbow is mostly originated by the coupling to states into the continuum. Besides coupling to bound and unbound states, also coupling to single and two neutron stripping can be of importance in describing the low-energy scattering of a halo nucleus. This has been investigated in [9, $10]$ and according to the authors it has a significant effect on the elastic scattering of ${ }^{6} \mathrm{He}$ and should not be neglected. Moreover, coupling effects of break-up or transfer depend upon nuclear structure properties of projectile and/or target as well as break-up thresholds or beam energies. Therefore drawing general conclusions about channel coupling effects on a limited sets of reactions induced by ${ }^{6} \mathrm{He}$ could be misleading (see $[11,12])$. Moreover, due to the complexity of modelling reactions with the $2 \mathrm{n}$-halo ${ }^{6} \mathrm{He}$ it would be of great help to have detailed elastic scattering data of 1n-halo systems, such as ${ }^{11} \mathrm{Be}$.

The only data published so far on elastic scattering and reaction mechanisms around the Coulomb barrier with neutron halo nuclei different than ${ }^{6} \mathrm{He}$, concerns the system ${ }^{11} \mathrm{Be}+{ }^{209} \mathrm{Bi}$ [13]. The authors used a fragmentation beam degraded in energy selecting, via a tagging procedure, beam energy bins having a $2 \mathrm{MeV}$ width. The quasi elastic cross-section, which included inelastic excitations up to $2.6 \mathrm{MeV}$, was measured. The TR cross-section extracted was found to be similar to the one of ${ }^{9} \mathrm{Be}+{ }^{209} \mathrm{Bi}$, measured by the same group [14]. The authors concluded that the break-up process has comparable strengths in both ${ }^{9,11} \mathrm{Be}$ nuclei and that the differences in binding energies and radii of the two Be isotopes do not play an important role. These conclusions are opposite to the ones reached with the ${ }^{6} \mathrm{He}$ beams by different authors. 
In this contribution we will report results concerning the measurement of elastic scattering angular distributions for the reactions ${ }^{9,10,11} \mathrm{Be}+{ }^{64} \mathrm{Zn}$ at the same center of mass energy, $E_{\text {c.m. }}=25.4 \mathrm{MeV}$, performed using for the first time high quality postaccelerated ${ }^{10,11} \mathrm{Be}$ beams delivered by the REX-ISOLDE facility (CERN). In the case of the halo ${ }^{11} \mathrm{Be}$ nucleus the transfer/break-up cross-section has been measured as well.

\section{Experimental Set-up}

The experiment with the stable ${ }^{9}$ Be beam was performed at Laboratori Nazionali del Sud (LNS) in Catania. The ${ }^{9}$ Be beam was delivered by the SMP 14MV Tandem of LNS and was impinging on a $550 \mu \mathrm{m} / \mathrm{cm}^{2}{ }^{64} \mathrm{Zn}$. The light charged particles emitted in the reaction have been detected using five $\Delta \mathrm{E}(\sim 10 \mu \mathrm{m}$ thick $)-\mathrm{E}(\sim 150 \mu \mathrm{m}$ thick $) \mathrm{Si}$ detector telescopes each consisting of a surface barrier detector. The five telescopes were placed on a rotating arm allowing the measurement of the elastic scattering angular distribution up to $110^{\circ}$.

Fig. 1 shows the apparatus used in the experiments with the radioactive ${ }^{10,11} \mathrm{Be}$ beams, performed at REX-ISOLDE (CERN). The detection system consisted of an array of $\Delta \mathrm{E}(\sim 50 \mu \mathrm{m}$ thick $)-\mathrm{E}(\sim 1500 \mu \mathrm{m}$ thick $)$ Si detector telescopes, each one with a surface of $50 \times 50 \mathrm{~mm}^{2}$, surrounding a thin ${ }^{64} \mathrm{Zn}$ target $\left(550 \mu \mathrm{g} / \mathrm{cm}^{2}\right.$ and 1000 $\mu \mathrm{g} / \mathrm{cm}^{2}$ for the ${ }^{10,11} \mathrm{Be}$ beams, respectively). The $\Delta \mathrm{E}$ stage consists of a Double sided Silicon Strip Detector (DsSSD) segmented in 16 both on the front side and on the rear side, allowing a 256 pixels for each detector. The E stage consists of a Silicon Single Pad detector. The detectors were placed very close to the target in order to have a large polar angle $\left(10^{\circ}<\theta<150^{\circ}\right)$ and solidangle coverage. The ${ }^{11} \mathrm{Be}$ beam energy is $29.8 \mathrm{MeV}$ with an average current of $10^{4} \mathrm{pps}$ whereas the ${ }^{10} \mathrm{Be}$ beam energy is $29.4 \mathrm{MeV}$ (in order to have the same center of mass energy of the ${ }^{11} \mathrm{Be}+{ }^{64} \mathrm{Zn}$ system) and an average current of $10^{6} \mathrm{pps}$.
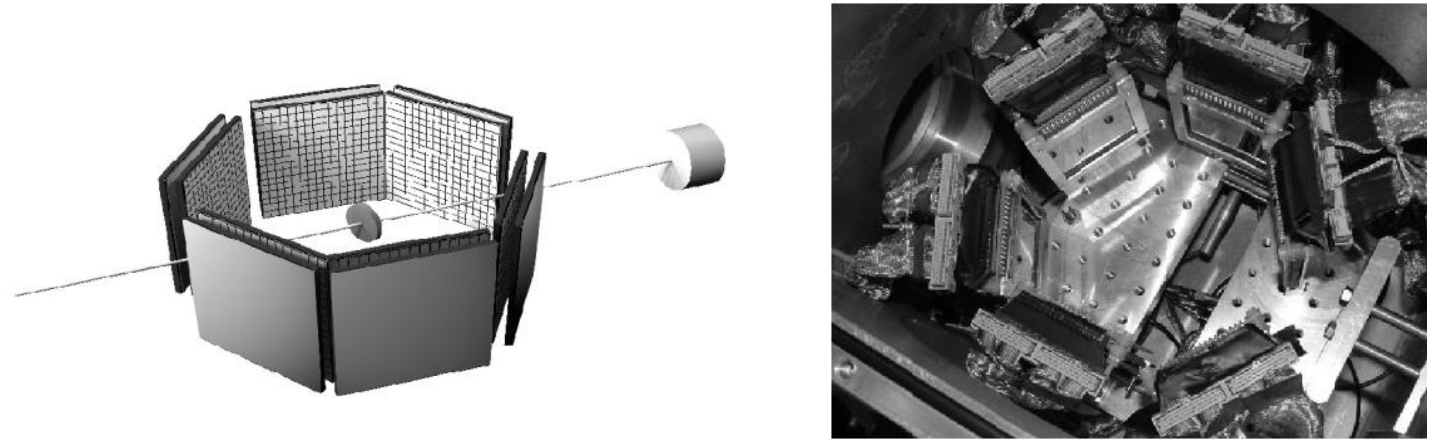

Figure 1. Schematic drawing of the 6 telescopes around the target (left) and picture of the detectors set-up inside the scattering chamber (right).

The effective solid angles covered by each detector pixel have been evaluated by using a Monte Carlo simulation code. However, due to the very compact geometry of the detection system, small variations of the beam position onto the target resulted in a large variation of the detector angles. Therefore, polar angle and solid angle determination has been verified by measuring the Rutherford scattering for the ${ }^{12} \mathrm{C}+{ }^{197} \mathrm{Au}$ reaction at $\mathrm{E}_{\mathrm{lab}}=28 \mathrm{MeV}$ as well as the elastic scattering angular distribution for the collision ${ }^{10} \mathrm{Be}+{ }^{197} \mathrm{Au}$ at $\mathrm{E}_{\mathrm{lab}}=29.4 \mathrm{MeV}$. With the adopted analysis procedure, 
the expected Rutherford behaviour of the cross-sections for the two reactions were obtained, indicating that the geometry was properly determined.

\section{Results and Discussion}

The ${ }^{9,11} \mathrm{Be}+{ }^{64} \mathrm{Zn}$ elastic scattering angular distributions at $\mathrm{E}_{\mathrm{c} . \mathrm{m} .}=25.4 \mathrm{MeV}$ are shown in fig. 2. In the case of ${ }^{11} \mathrm{Be}+{ }^{64} \mathrm{Zn}$ the cross-section includes also the ${ }^{11} \mathrm{Be}$ first excited states. However, according to OM calculations, the contribution of inelastic scattering is very small at all measured angles. From fig. 2 one can clearly see that for ${ }^{11} \mathrm{Be}$ induced scattering a reduction of the elastic cross-section with respect to the weakly bound ${ }^{9} \mathrm{Be}$ induced scattering is present both in the rainbow region as well as at large angles.

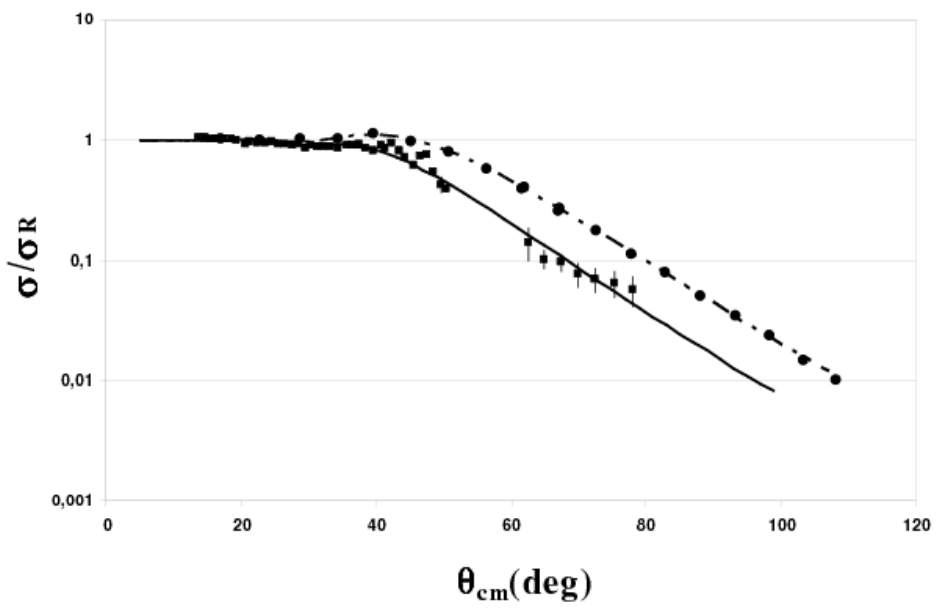

Figure 2. ${ }^{9} \mathrm{Be}+{ }^{64} \mathrm{Zn}$ (full circles) and ${ }^{11} \mathrm{Be}+{ }^{64} \mathrm{Zn}$ (full squares) elastic scattering angular distributions at $\mathrm{E}_{\mathrm{c} . \mathrm{m}}=25.4 \mathrm{MeV}$. The lines represent the results of the corresponding optical model fits.

The ${ }^{9,10,11} \mathrm{Be}+{ }^{64} \mathrm{Zn}$ elastic scattering data were analyzed within the $\mathrm{OM}$ using the code PTOLEMY [15]. For the ${ }^{9,10} \mathrm{Be}$ induced scattering a Wood-Saxon (W-S) form was used to describe the real and the imaginary part of the optical potential. In the ${ }^{11} \mathrm{Be}$ case, in order to take into account the halo features and the effect of the break-up channel on elastic scattering, a surface term was added to the volume part of the imaginary potential. In [16], in order to study break-up effects on elastic-scattering induced by ${ }^{11} \mathrm{Be}$, the imaginary part of the optical potential due to break-up (i.e. the surface term) was calculated analytically. The calculated surface term, which has an exponential shape, is responsible for peripheral reactions like transfer or break-up. The shape of this term and its parameters depend only on the halo neutron break-up probability. The authors found that the addition of such a surface potential is important for a reduction of the elastic cross-section of the halo ${ }^{11} \mathrm{Be}$ nucleus. Therefore, following [16], we used a similar procedure to deduce the optical potential in the case of ${ }^{11} \mathrm{Be}+{ }^{64} \mathrm{Zn}$. In the present case, all terms of the optical potential are extracted phenomenologically from a fit of the elastic scattering angular distributions. The fit was performed using as the volume part of the real and imaginary $\mathrm{W}-\mathrm{S}$ potential, the OM parameters obtained from the fit of the elastic scattering of the ${ }^{10} \mathrm{Be}$, since it represents the core of the halo ${ }^{11} \mathrm{Be}$ 
nucleus. The shape of the surface potential is a W-S derivative type. The effect of such term is to reduce the elastic scattering cross-section at all measured angles and in particular we have found that in order to reproduce the behaviour of the elastic crosssection at the rainbow a very large imaginary surface diffuseness parameter, $\mathrm{a}_{s i}=3.5 \mathrm{fm}$, must be used. A similar result was also obtained in [16], where a diffuseness parameter of $3.2 \mathrm{fm}$ was found. The large diffuseness is an evidence that in the case of ${ }^{11} \mathrm{Be}$ the coupling to break-up states can occur beyond the distance of closest approach, causing the suppression of the elastic cross-section at the rainbow and reflecting the long range of the absorptive surface potential.

Along with the optical potential parameters, the TR cross-sections were extracted. We obtained the following values: $\sigma_{R}=1080 \pm 50 \mathrm{mb}$ for ${ }^{9} \mathrm{Be}$ and $\sigma_{R}=$ $2250 \pm 250 \mathrm{mb}$ for ${ }^{11} \mathrm{Be}$. Actually, the TR cross-section for the ${ }^{11} \mathrm{Be}$ case is double than the one extracted for the ${ }^{9} \mathrm{Be}$ case. In order to better understand the contribution due to direct processes to the large TR cross-section observed in the ${ }^{11} \mathrm{Be}$ induced collision the transfer and break-up events were also analyzed. The corresponding cross-section is $\sigma_{\text {trans/b-up }}=1100 \pm 150 \mathrm{mb}$. Therefore, by comparing this cross-section with the TR crosssection, deduced from the fit on the ${ }^{11} \mathrm{Be}$ scattering data, almost $50 \%$ of the TR crosssection is associated to transfer and break-up processes. These results are in general agreement with the results obtained with ${ }^{6} \mathrm{He}$ beam (e.g. $\left.[1,2]\right)$ but disagree with the previously published data of ${ }^{11} \mathrm{Be}$ [13].

\section{References}

1. E. Aguilera et al., Phys. Rev. C 63, 061603(R) (2001).

2. A. Di Pietro et al., Phys. Rev. C 69, 044613 (2004).

3. R. Raabe et al., Nature 431, 823 (2004).

4. Yu.E. Penionzhkevich et al., Phys. Rev. Lett. 96, 162701(2006).

5. A. M. Sanchez-Benitez, et al., Nucl. Phys. A 803, 30 (2008).

6. J.P. Bychowski et al., Phys. Rev. Lett. 59626 (2004).

7. A. Moro, et al., Phys. Rev. C 75, 064607 (2007).

8. M. Rodriguez-Gallardo, et al., Phys. Rev. C 77, 064609 (2008).

9. N.Keeley and N. Alamanos, Phys. Rev. C 77, 054602 (2008).

10. A. Chatterjee, et al., Phys. Rev. Lett. 101, 032701 (2008).

11. N. Keeley et al., Prog. Part. Nuc. Phys. 59, 579 (2007).

12. N. Keeley et al., Prog. Part. Nuc. Phys. 63, 396 (2009).

13. Mazzocco et al. Eur. Phys. J. Special Topics 150, 37 (2007).

14. C. Signorini, et al., Nucl. Phys. A 701, 23c (2002).

15. M. J. Rhoades-Brown et al., Phys. Rev. C 21, 2417 (1980).

16. A. Bonaccorso, F. Carstoiu Nucl. Phys. A 706322 (2002). 